\title{
Prolonged Hyponatremia after Pituitary Surgery: A Case Report and Literature Review
}

\author{
Meriem Yazidi ${ }^{1}$, Marwa Ben Jemaa ${ }^{2}$, Ibtissem Oueslati ${ }^{3}$, Fatma Chaker ${ }^{4}$, Melika Chihaoui ${ }^{5}$
}

\begin{abstract}
Hyponatremia is commonly seen after pituitary surgery. It generally occurs within the first week after surgery and is usually moderate and asymptomatic. However, it may be severe and prolonged in some cases. Different etiologies can explain this hyponatremia. The syndrome of inappropriate antidiuretic hormone secretion (SIADH) is one of the most common etiologies, but other differential diagnoses must be ruled out before making this diagnosis. In this article, we present a case of prolonged hyponatremia caused by SIADH occurring after transsphenoidal surgery for a non-functioning pituitary macroadenoma. We revise the different etiologies of hyponatremia following pituitary surgery and their treatment.

Keywords: Cerebral salt-wasting syndrome, Hyponatremia, Pituitary gland, Syndrome of inappropriate secretion of antidiuretic hormone, Transsphenoidal surgery.

World Journal of Endocrine Surgery (2020): 10.5005/jp-journals-10002-1305
\end{abstract}

\section{BACKGROUND}

Hydroelectrolytic disorders are commonly seen after pituitary surgery. ${ }^{1-3}$ Transient central diabetes insipidus (DI) is the most known hydroelectrolytic disorder following pituitary surgery and is usually responsible for transient hypernatremia. Transient hyponatremia is also frequently encountered after pituitary surgery. Different etiologies can explain this hyponatremia. The syndrome of inappropriate secretion of antidiuretic hormone (SIADH) is one of the most common etiologies, but other differential diagnoses must be ruled out before making this diagnosis. Hyponatremia after pituitary surgery is generally transient and poorly or no symptomatic. However, in some cases, it can be severe and prolonged resulting in higher postoperative morbidity and prolonged hospitalization. ${ }^{2,4,5}$ Herein, we report a case of prolonged hyponatremia related to SIADH, occurring after transsphenoidal surgery for a non-functioning pituitary adenoma and we review the different etiologies of hyponatremia occurring after pituitary surgery and their appropriate treatment.

\section{Case Description}

A 45-year-old woman was referred from the neurosurgery department to the endocrinology department for prolonged hyponatremia following pituitary surgery. She underwent an endoscopic transsphenoidal surgery 1 month earlier for a nonfunctioning pituitary macroadenoma revealed by an optochiasmatic syndrome and secondary amenorrhea. The patient developed an acute and persistent postoperative hyponatremia which was initially attributed to a secondary adrenocortical insufficiency. She was treated with high doses of hydrocortisone and isotonic saline infusion without improvement of her hyponatremia which reached a nadir in the eleventh postoperative day (Fig. 1).

On admission, the patient was complaining of persistent headaches. The physical examination found a weight of $55 \mathrm{~kg}$ (stable), a lying and standing blood pressure at $120 / 80 \mathrm{~mm} \mathrm{Hg}$, a heart rate of 80 beats/minute. No symptoms of volume depletion were present. Laboratory tests showed hyponatremia ( $\mathrm{Na}^{+}: 122$ $\mathrm{mmol} / \mathrm{L}$ ) with low plasmatic osmolality (256 $\mathrm{mOsmol} / \mathrm{L})$ and
${ }^{1-5}$ Department of Endocrinology, Universite de tunis el manar, Faculte de Medecine de Tunis, La Rabta Hospital, Tunis, Tunisia

Corresponding Author: Meriem Yazidi, Department of Endocrinology, Universite de tunis el manar, Faculte de Medecine de Tunis, La Rabta Hospital, Tunis, Tunisia, Phone: +21698266061, e-mail: meriemyazidi@ gmail.com

How to cite this article: Yazidi M, Ben Jemaa M, Oueslati l, et al. Prolonged Hyponatremia after Pituitary Surgery: A Case Report and Literature Review. World J Endoc Surg 2020;12(3):128-131.

Source of support: Nil

Conflict of interest: None

elevated natriuresis ( $78 \mathrm{mmol} / 24$ hours). Serum urea, albumin, and hematocrit levels were normal. The thyroid function was normal on $50 \mu \mathrm{g}$ of L-thyroxine. The diagnosis of SIADH was held and the patient was put on water restriction $(500 \mathrm{~mL} /$ day). Sodium level

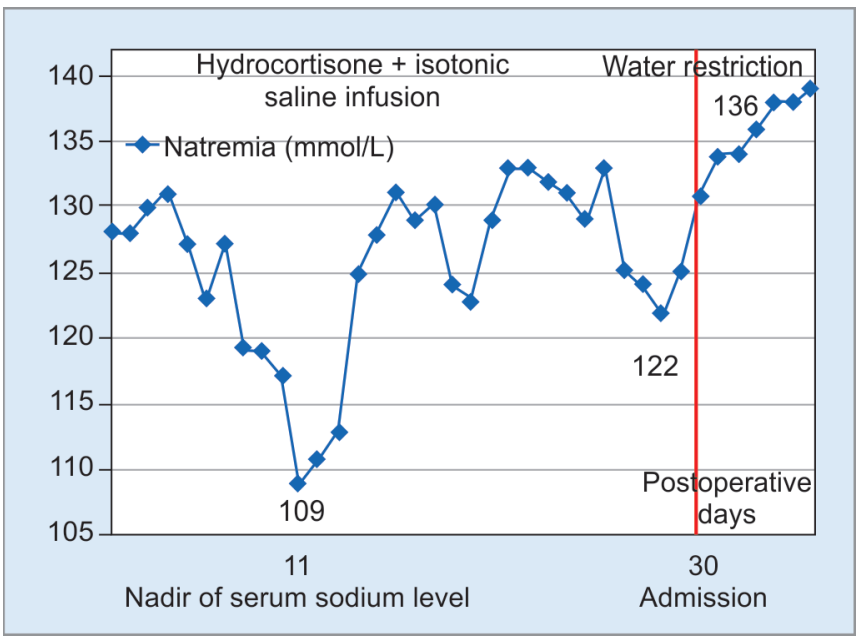

Fig. 1: Evolution of patient's serum sodium levels after transsphenoidal surgery

( ) Jaypee Brothers Medical Publishers. 2020 Open Access This article is distributed under the terms of the Creative Commons Attribution 4.0 International License (https://creativecommons.org/licenses/by-nc/4.0/), which permits unrestricted use, distribution, and non-commercial reproduction in any medium, provided you give appropriate credit to the original author(s) and the source, provide a link to the Creative Commons license, and indicate if changes were made. The Creative Commons Public Domain Dedication waiver (http://creativecommons.org/publicdomain/zero/1.0/) applies to the data made available in this article, unless otherwise stated. 
increased progressively reaching normal level the fifth day of water restriction (Fig. 1).

\section{Discussion}

\section{Prevalence and Time to Onset of Hyponatremia after Pituitary Surgery}

Hyponatremia is a common complication of pituitary surgery. It occurs in $16-38 \%$ of patients. ${ }^{4-8}$ Fisher described a classic triphasic trend of hydroelectrolytic disturbances after pituitary surgery in which the first phase of central DI is initially observed, followed by a phase of transient hyponatremia related to SIADH 1 week later and a final phase of permanent or transient central DI. This classic triphasic evolution is actually observed in only $1-2 \%$ of cases. ${ }^{9}$ Hyponatremia usually occurs between the fifth and ninth day after surgery but it can also appear earlier in the first 24 hours or later when the patient has been discharged..$^{4-6}$ Hence, there is a need to educate the patient about the signs of hyponatremia and advice consult if symptoms appear. In the reported case, the patient developed hyponatremia on the first postoperative day, which progressively worsened and reached a nadir $\left(\mathrm{Na}^{+}=109 \mathrm{mEq} / \mathrm{L}\right)$ on the 11th day postoperatively. This hyponatremia persisted for $>30$ days after the surgery.

\section{Risk Factors of Hyponatremia after Pituitary Surgery}

Many studies showed that advanced age, female gender, prior development of $\mathrm{DI}^{10}$ preoperative hypopituitarism, ${ }^{11}$ and $\mathrm{ACTH}$ secreting adenoma ${ }^{4}$ may be predictive factors for the development of postoperative hyponatremia. The relationship between tumor size and hyponatremia risk is still controversial., ${ }^{711}$

\section{Severity and Clinical Manifestations of Hyponatremia}

In a study of 64 patients who developed hyponatremia after pituitary surgery, Barber et al. found that mild forms (serum sodium 131-134 mEq/L) were the most common (51.6\%) followed in frequency by moderate hyponatremia (serum sodium 125-130 $\mathrm{mEq} / \mathrm{L}$ ) in $30.6 \%$ of the cases then severe hyponatremia (serum sodium $<125 \mathrm{mEq} / \mathrm{L}$ ) in $17.7 \%$ of them. ${ }^{7}$

The clinical manifestations of hyponatremia depend on its severity and its speed of development. Most often this hyponatremia is asymptomatic resolving spontaneously within a few days. It can therefore go unnoticed. Hyponatremia becomes symptomatic if it is deep ( $<120 \mathrm{mmol} / \mathrm{L})$ and in case of rapid onset

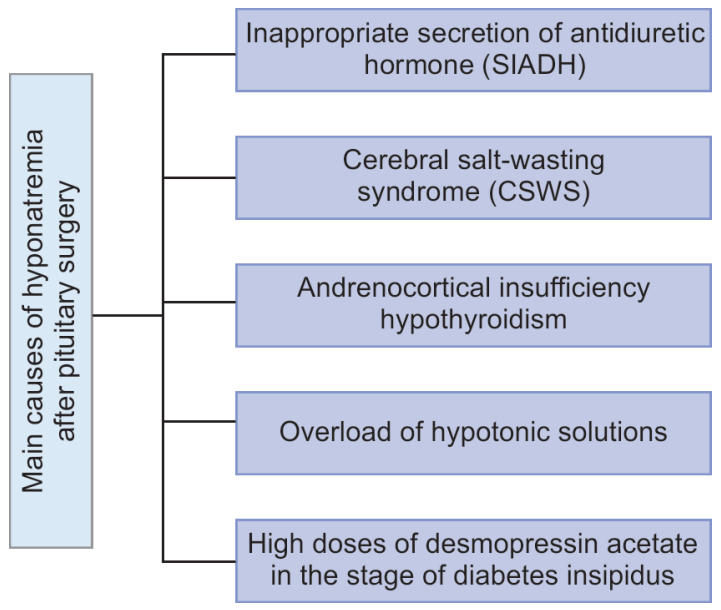

Fig. 2: Etiologies of hyponatremia after pituitary surgery
( $<48$ hours). Symptoms reflect the cerebral edema due to plasmatic hypoosmolality: headache, nausea, vomiting, abdominal pain, malaise, agitation, confusion, coma, etc. ${ }^{12}$ In the case reported it manifested only by headache.

\section{Etiologies}

Syndrome of inappropriate secretion of antidiuretic hormone and cerebral salt-wasting syndrome (CSWS) are the main causes of hyponatremia following pituitary surgery, but hyponatremia can also be related to adrenocortical insufficiency or hypothyroidism. Hyponatremia may also be caused by an overload of hypotonic solutions received during the perioperative period or by high doses of desmopressin acetate given during the stage of DI (Fig. 2). After having ruled out these two latter iatrogenic causes of hyponatremia and correctly substituted the eventual corticotropin and thyrotropin deficiencies, distinguishing between the diagnoses of SIADH and CSWS can be difficult because these two entities have many clinical and biological similarities. However, it is essential to differentiate between these two disorders because the management of hyponatremia is different. ${ }^{4,8,13-16}$

\section{SIADH}

Surgical manipulation of the posterior pituitary induces a release of $\mathrm{ADH}$. As the serum osmolality and blood volume are normal, this secretion of $A D H$ is considered inappropriate. This results in water retention and expansion of extracellular volume which induces an increase in glomerular filtration rate with a decrease in renal sodium reabsorption. Hence, urinary sodium excretion is increased and the blood volume remains normal or slightly increased. ${ }^{4,10,12,17,18}$ Syndrome of inappropriate secretion of antidiuretic hormone is therefore characterized by hyponatremia associated with hypernatriuresis and normal or slightly increased blood volume (Fig. 3).

Syndrome of inappropriate secretion of antidiuretic hormone diagnosis is based on Barter and Schwartz criteria (Table 1).

\section{Cerebral Salt-wasting Syndrome}

Cerebral salt-wasting syndrome is a volume-depleted state caused by excessive natriuresis occurring in patients with intracranial disease that leads to hyponatremia. ${ }^{19}$ This syndrome is also involved

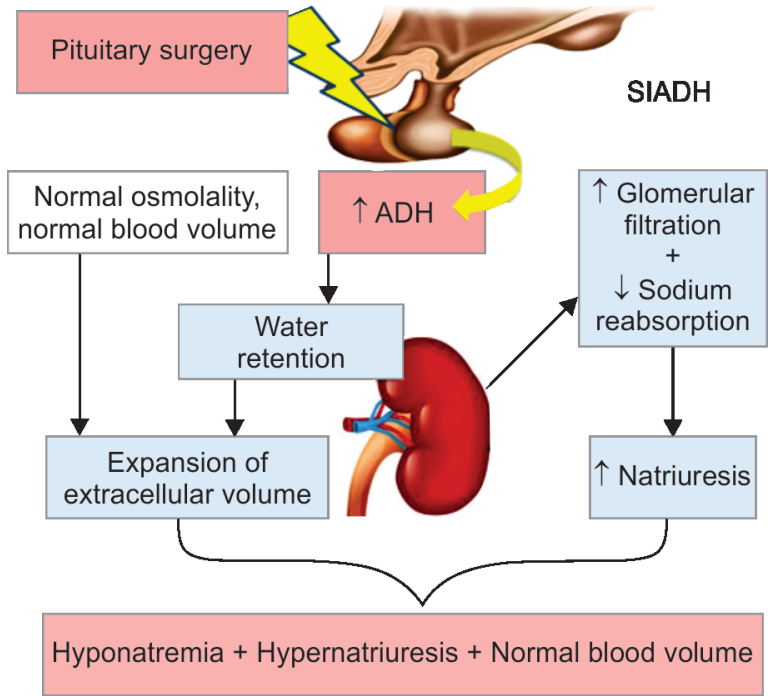

Fig. 3: Pathophysiology of syndrome of inappropriate secretion of antidiuretic hormone (SIADH) 
in many hyponatremias occurring after pituitary surgery. ${ }^{4,13,19,20}$ The pathophysiology of this disorder remains unclear but hypotheses incriminate some natriuretic factors such as atrial natriuretic peptide (ANP), brain natriuretic peptide (BNP), C-type natriuretic peptide, and ouabain-like peptide (OLP) associated with decreased sympathetic input to the kidney. These natriuretic factors reduce effective blood volume by increasing natriuresis. This volume depletion stimulates $A D H$ secretion but this secretion is not considered inappropriate as in SIADH. ${ }^{7,12-14,17,20}$

The decrease in the activity of the sympathetic nervous system at the renal level increases the hypernatriuresis by direct and indirect action via a decrease in the activity of the reninangiotensin-aldosterone system despite hypovolemia. Cerebral salt-wasting syndrome is therefore characterized by hyponatremia associated with hypernatriuresis and hypovolemia (Fig. 4).

\section{Differential Diagnosis between SIADH and CSWS}

Assessment of extracellular volume makes it possible to differentiate between the two syndromes: CSWS is characterized by decreased plasma volume with signs of dehydration while SIADH is characterized by euvolemia or mild hypervolemia. ${ }^{1,4,15,17,19,21,22}$ The extracellular volume status is however difficult to assess. Table 2

Table 1: Diagnostic criteria of the syndrome of inappropriate secretion of antidiuretic hormone (SIADH) $)^{4,12}$
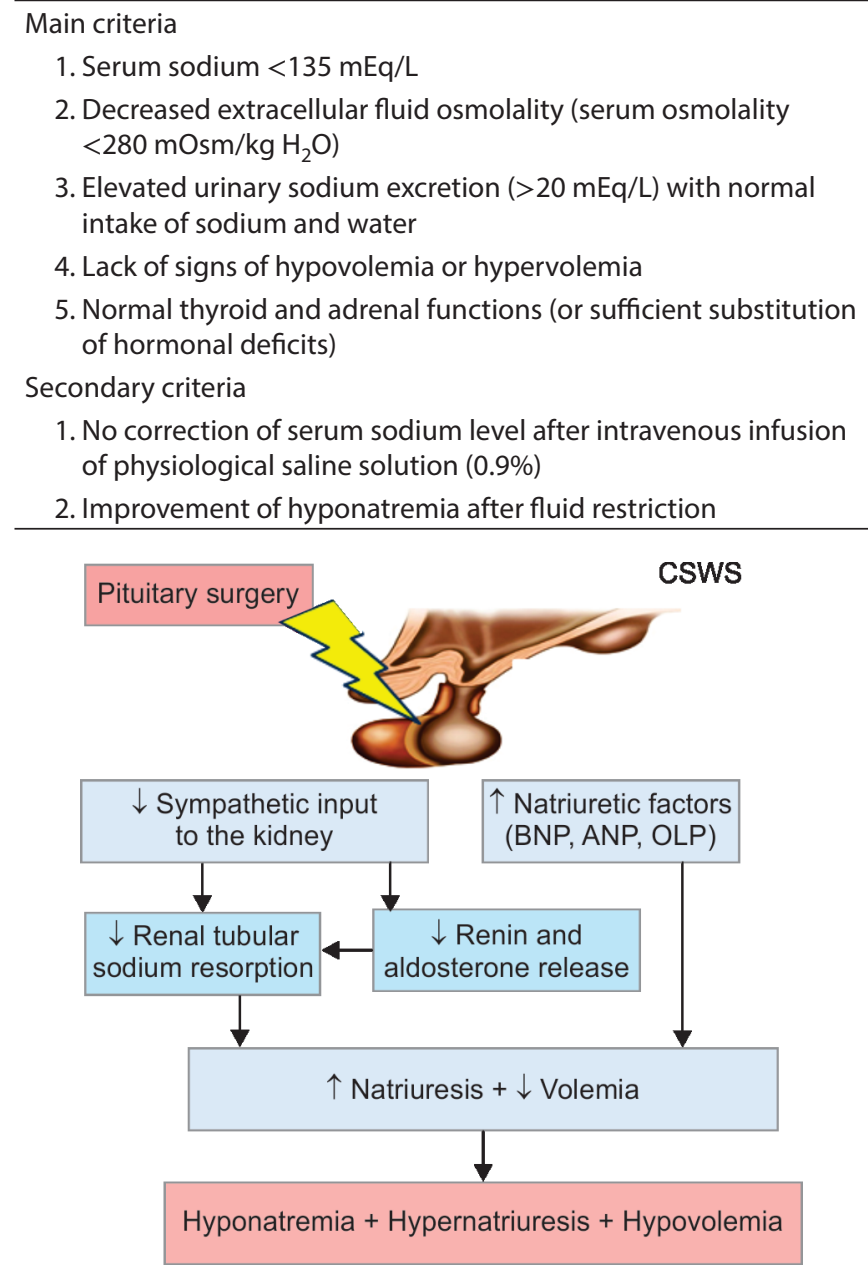

Fig. 4: Pathophysiology of cerebral salt-wasting syndrome (CSWS); ANP (atrial natriuretic peptide); BNP (brain natriuretic peptide); OLP (oubain-like peptide) shows the most relevant clinical and biological parameters in clinical practice that allow the distinction between the two syndromes by appreciating extracellular volume status.

In the reported case, the diagnosis of SIADH was based on the absence of clinical and biological signs of extracellular volume depletion (stable weight, normal heart rate, absence of orthostatic hypotension, low serum osmolality, normal serum urea, albumin, and hematocrit levels) and the normalization of serum sodium after water restriction.

\section{Management of Postoperative Hyponatremia}

The management of hyponatremia occurring in the context of pituitary surgery includes:

- The elimination of iatrogenic causes.

- The adequate substitution of eventual associated corticotropin and thyrotropin deficiencies.

- The evaluation of the severity and speed of onset of hyponatremia.

- The distinction between SIADH and CSWS.

It should not be forgotten that a too rapid correction of hyponatremia may be complicated by osmotic demyelination, which prognosis is often severe. Thus, asymptomatic and chronic hyponatremia (onset $>48$ hours) higher than $115 \mathrm{mmol} / \mathrm{L}$ is not a therapeutic emergency. Only acute and poorly tolerated hyponatremia with neurological signs should be immediately corrected in a neurosurgical intensive care unit. In this case, it is necessary to rapidly increase the natremia with hypertonic saline solution (at 3\%) to reduce cerebral edema and prevent cerebral engagement. ${ }^{18}$ However, in chronic and well-tolerated hyponatremia, it is essential to distinguish between SIADH and CSWS before treating. ${ }^{4,6,15}$ As the pathophysiology in SIADH lies in inappropriate reabsorption of free water, the gold standard treatment for SIADH consists of fluid restriction. ${ }^{4,18}$ In contrast, CSWS treatment is salt and fluid replacement. Either isotonic or hypertonic saline solutions are effective. ${ }^{7,23}$ In SIADH, the fluid restriction rate depends on sodium concentration. Casulari et al. have suggested the following rates according to serum sodium levels: $<1,200 \mathrm{~mL} /$ day if the natremia is between 130 and $134 \mathrm{mEq} / \mathrm{L},<800 \mathrm{~mL} /$ day between 126 and $130 \mathrm{mEq} / \mathrm{L}$, and $<600 \mathrm{~mL} /$ day if natremia is under $125 \mathrm{mEq} / \mathrm{L}^{22}$ Fluid intake was limited to $500 \mathrm{~mL} /$ day in the reported case. In CSWS, sodium replacement can be performed by an infusion of physiological saline solution $(0.9 \%)$ if the natremia

Table 2: Differential diagnosis between SIADH and CSWS in clinical practice

\begin{tabular}{lcc}
\hline & CSWS & SIADH \\
\hline Clinical parameters & & \\
Weight & $\downarrow$ & $\uparrow$ or $\leftrightarrow$ \\
Tachycardia & Present & Absent \\
Orthostatic hypotension & Present & Absent \\
Urinary volume & $\leftrightarrow$ or $\uparrow$ & $\leftrightarrow$ or $\downarrow$ \\
Biological parameters & & \\
Serum osmolality & $\leftrightarrow$ or $\uparrow$ & $\downarrow$ \\
Urea & $\uparrow$ & $\leftrightarrow$ or $\downarrow$ \\
Hematocrit & $\uparrow$ & $\leftrightarrow$ \\
Serum protein level & $\uparrow$ & $\leftrightarrow$ \\
\hline
\end{tabular}

CSWS, cerebral salt-wasting syndrome; SIADH, inappropriate secretion of antidiuretic hormone syndrome; $\downarrow$, decreased; $\uparrow$, increased; $\leftrightarrow$, unchanged 
is between 125 and $130 \mathrm{mEq} / \mathrm{L}$ and by hypertonic saline solution if the natremia is under $120 \mathrm{mEq} / \mathrm{L}$. Chronic hyponatremia should not be corrected by $>10 \mathrm{mEq} /$ day to avoid osmotic centropontine demyelination and treatment is not required if natremia is $>130$ $\mathrm{mEq} / \mathrm{L} .{ }^{4,18}$ Fluid restriction may worsen hyponatremia in patients with CSWS. It is therefore essential to make the correct diagnosis before starting the treatment. $4,13,14,18,20$

\section{Conclusion}

Hyponatremia is commonly observed after pituitary surgery. It usually occurs within the first week after surgery and is usually moderate and asymptomatic. However, it may be severe in some cases and may occur after the patient has been discharged justifying the importance of educating the patient about the clinical signs of hyponatremia. The management depends on the etiology and must take into account the severity and speed of onset of hyponatremia. Acute, severe, and symptomatic hyponatremia is a life-threatening emergency that must be managed in a neurosurgical intensive care unit. Chronic hyponatremia must be corrected slowly to avoid osmotic demyelination syndrome. After having ruled out the iatrogenic causes of hyponatremia and correctly substituted the eventual corticotropin and thyrotropin deficiencies, distinguishing between the diagnoses of SIADH and CSWS is crucial for determining the appropriate treatment. Water restriction is indicated in the case of SIADH and slow saline infusion in the case of CSWS.

\section{Statement of Ethics}

The patient gave her written consent for the data used in the manuscript.

\section{References}

1. Verbalis JG. Management of disorders of water metabolism in patients with pituitary tumors. Pituitary 2002;5(2):119-132. DOI: 10.1023/a:1022368615879.

2. Sata A, Hizuka N, Kawamata T, et al. Hyponatremia after transsphenoidal surgery for hypothalamo-pituitary tumors. Neuroendocrinology 2006;83(2):117-122. DOI: 10.1159/000094725.

3. Kiran Z, Sheikh A, Momin SNA, et al. Sodium and water imbalance after sellar, suprasellar, and parasellar surgery. Endocrine practice 2017;23(3):309-317. DOI: 10.4158/EP161616.OR.

4. Guerrero R, Pumar A, Soto A, et al. Early hyponatraemia after pituitary surgery: cerebral salt-wasting syndrome. Eur J Endocrinol 2007;156(6):611-616. DOI: 10.1530/EJE-06-0659.

5. Taylor SL, Tyrrell JB, Wilson CB. Delayed onset of hyponatremia after transsphenoidal surgery for pituitary adenomas. Neurosurgery 1995;37(4):649-653. DOI: 10.1227/00006123-199510000-00007.

6. Sane T, Rantakari K, Poranen A, et al. Hyponatraemia after transsphenoidal surgery for pituitary tumor. J Clin Endocrinol Metabol 1994;79(5):1395-1398. DOI: 10.1210/jcem.79.5.7962334.

7. Barber SM, Liebelt BD, Baskin DS. Incidence, etiology and outcomes of hyponatremia after transsphenoidal surgery: experience with
344 consecutive patients at a single tertiary center. J Clin Med 2014;3(4):1199-1219. DOI: 10.3390/jcm3041199.

8. Tomita Y, Kurozumi K, Inagaki K, et al. Delayed postoperative hyponatremia after endoscopic transsphenoidal surgery for pituitary adenoma. Acta Neurochir (Wien) 2019;161(4):707-715. DOI: 10.1007/ s00701-019-03818-3.

9. Fisher C, Ingram WR. The effect of interruption of the supraopticohypophyseal tracts on the antidiuretic, pressor and oxytocic activity of the posterior lobe of the hypophysis. Endocrinology 1936;20(6):762-768. DOI: 10.1210/endo-20-6-762.

10. Zada G, Liu CY, Fishback D, et al. Recognition and management of delayed hyponatremia following transsphenoidal pituitary surgery. J Neurosurg 2007;106(1):66-71. DOI: 10.3171/jns.2007.106.1.66.

11. Jahangiri A, Wagner J, Tran MT, et al. Factors predicting postoperative hyponatremia and efficacy of hyponatremia management strategies after more than 1000 pituitary operations. J Neurosurg 2013;119(6):1478-1483. DOI: 10.3171/2013.7.JNS13273.

12. Cuesta M, Hannon MJ, Thompson CJ. Diagnosis and treatment of hyponatraemia in neurosurgical patients. Endocrinol Nutr 2016;63(5):230-238. DOI: 10.1016/j.endonu.2015.12.007.

13. Palmer BF. Hyponatraemia in a neurosurgical patient: syndrome of inappropriate antidiuretic hormone secretion versus cerebral salt wasting. Nephrol Dial Transplant 2000;15(2):262-268. DOI: 10.1093/ ndt/15.2.262

14. Cui H, He G, Yang S, et al. Inappropriate antidiuretic hormone secretion and cerebral salt-wasting syndromes in neurological patients. Front Neurosci 2019;13:1170. DOI: 10.3389/fnins.2019. 01170.

15. Rahman M, Friedman WA. Hyponatremia in neurosurgical patients: clinical guidelines development. Neurosurgery 2009;65(5):925-935. DOI: 10.1227/01.NEU.0000358954.62182.B3.

16. Olson BR, Rubino D, Gumowski J, et al. Isolated hyponatremia after transsphenoidal pituitary surgery. J Clin Endocrinol Metab 1995;80(1):85-91. DOI: 10.1210/jcem.80.1.7829644.

17. Tudor RM, Thompson CJ. Posterior pituitary dysfunction following traumatic brain injury: review. Pituitary 2019;22(3):296-304. DOI: 10.1007/s11102-018-0917-z.

18. Fraser JF, Stieg PE. Hyponatremia in the neurosurgical patient: epidemiology, pathophysiology, diagnosis, and management. Neurosurgery 2006;59(2):222-229. DOI: 10.1227/01. NEU.0000223440.35642.6E.

19. Harrigan MR. Cerebral salt wasting syndrome: a review. Neurosurgery 1996;38(1):152-160. DOI: 10.1097/00006123-199601000-00035.

20. Atkin SL, Coady AM, White MC, et al. Hyponatraemia secondary to cerebral salt wasting syndrome following routine pituitary surgery. Eur J Endocrinol 1996;135(2):245-247. DOI: 10.1530/eje.0.1350245.

21. Leonard J, Garrett RE, Salottolo K, et al. Cerebral salt wasting after traumatic brain injury: a review of the literature. Scand $J$ Trauma Resusc Emerg Med 2015;23(1):98. DOI: 10.1186/s13049-0150180-5.

22. Casulari LA, Costa KN, Albuquerque R, et al. Differential diagnosis and treatment of hyponatremia following pituitary surgery. J Neurosurg Sci 2004;48(1):11-18.

23. Suarez Jl, Qureshi Al, Parekh PD, et al. Administration of hypertonic (3\%) sodium chloride/acetate in hyponatremic patients with symptomatic vasospasm following subarachnoid hemorrhage. J Neurosurg Anesthesiol 1999;11(3):178-184. DOI: 10.1097/00008506$199907000-00004$. 